\title{
Molecular mechanisms of lung-specific toxicity induced by epidermal growth factor receptor tyrosine kinase inhibitors (Review)
}

\author{
SEIICHIRO SAKAO and KOICHIRO TATSUMI \\ Department of Respirology (B2), Graduate School of Medicine, Chiba University, Chuo-ku, Chiba 260-8670, Japan
}

Received May 17, 2012; Accepted August 14, 2012

DOI: $10.3892 / \mathrm{ol} .2012 .872$

\begin{abstract}
Lung-specific toxicity induced by epidermal growth factor receptor (EGFR) tyrosine kinase inhibitors (TKIs) for the treatment of non-small cell lung cancer (NSCLC) has emerged as a critical side-effect. Although the clinical features of the pulmonary side-effects of TKIs have been characterized, the details of the molecular mechanisms in the development of this lung-specific toxicity remain to be elucidated. EGFR-dependent epithelial regeneration and restoration plays an important role in the recovery process from lung injury. The lung comprises a unique environment where epithelial cells are exposed to internal agents in the systemic circulation and to airborne particles through the mouth and nose. This unique environment may also be associated with the development of lung-specific toxicity induced by EGFR-TKIs. Therefore, the aim of this review was to provide further insight into the molecular mechanisms of lung-specific toxicity in the context of treatment with EGFR-TKIs.
\end{abstract}

\section{Contents}

1. Introduction

2. Tyrosine kinase inhibitors (TKIs)

3. The pathogenesis of lung fibrosis and epidermal growth factor receptor (EGFR)

4. Lung metabolic function

5. Conclusion

Correspondence to: $\mathrm{Dr}$ Seiichiro Sakao, Department of Respirology (B2), Graduate School of Medicine, Chiba University, 1-8-1 Inohana, Chuo-ku, Chiba 260-8670, Japan

E-mail: sakaos@faculty.chiba-u.jp

Key words: epidermal growth factor receptor, tyrosine kinase inhibitors, lung fibrosis

\section{Introduction}

The lung-specific toxicity induced by epidermal growth factor receptor (EGFR) tyrosine kinase inhibitors (TKIs) for the treatment of non-small cell lung cancer (NSCLC) has emerged as a critical side-effect (1-7). Although the clinical features of the pulmonary side-effects of TKIs have been characterized (8-10), the details of the molecular mechanisms in the development of this lung-specific toxicity remain to be elucidated. The aim of this review was to provide further insight into the molecular mechanisms of lung-specific toxicity in the context of treatment with EGFR-TKIs.

\section{Tyrosine kinase inhibitors (TKIs)}

Recent advances in molecular targeted therapy have demonstrated improved response rates and progression free survival, particularly with TKIs which may act on the EGFR, in NSCLC patients $(11,12)$. Tyrosine kinases are enzymes that transfer the terminal phosphate from ATP to tyrosine residues in order to activate them, and the signaling pathways through receptor tyrosine kinases (RTKs) have been demonstrated to play a role in cancer development. Therefore, different types of TKIs have been developed and used in the treatment of cancer. EGFR-TKIs were adapted for lung cancer treatment since EGFR is frequently overexpressed and occasionally mutated in NSCLC cells (13-15).

There are two types of TKIs: non-receptor TKIs and receptor TKIs. The former TKIs bind to the active site of a non-receptor tyrosine kinase to prevent phosphorylation. Imatinib is a non-receptor TKI, which is used for the treatment of chronic myelogenous leukemia (CML), gastrointestinal stromal tumors (GISTs) and other diseases (16). The latter TKIs are able to bind to the active site of a RTK and some may exhibit a selective inhibitory effect on a certain RTK. Erlotinib and gefitinib are selective inhibitors of the EGFR tyrosine kinase domain. Erlotinib is used for the treatment of pancreatic carcinomas and NSCLC, while gefitinib is used for the treatment of NSCLC (9). A number of TKIs targeting several cancers have been approved for clinical use, and numerous newly developed TKIs are currently undergoing clinical trials (17).

Lung-specific toxicities induced by treatment with TKIs are rare (8). However, lung fibrosis associated with 
EGFR-TKIs is the most prominent in specific toxicity (9). The incidence of lung fibrosis ranges from 0.2 to $1.1 \%$ in erlotinib-treated patients and from 0.38 to $2.0 \%$ in gefitinibtreated patients (9). Additionally, a higher incidence of gefitinib-induced lung fibrosis is observed in Japanese patients (1). The predictive risk factors for the development of lung fibrosis include male gender, smoking history and antecedent lung fibrosis (4).

Randomized controlled trials (RCTs) of a new TKIs (originally BIBF 1120) assessing lung fibrosis were recently launched (18). This TKI is a per os active fibroblast growth factor receptor (FGFR), vascular endothelial growth factor receptor (VEGFR) and platelet-derived growth factor receptors (PDGFR)-TKI that is supposed to be effective against lung fibrosis since certain tyrosine kinase pathways have been demonstrated to be included in the development of lung fibrotic lesions (18). However, lung fibrosis is one of the adverse effects induced by an active selective EGFR-TKI for the treatment of NSCLC (8-10). These results indicate that only the EGFR pathway in the tyrosine kinase activities may account for the lung fibrosis in the patients treated with these drugs. Additionally, the EGFR-dependent pathway may be essential for the maintenance of the lung parenchyma consisting of the alveolar epithelium.

\section{The pathogenesis of lung fibrosis and epidermal growth factor receptor (EGFR)}

A unifying mechanism that is able to completely explain all fibrotic lung diseases remains to be elucidated. The original hypothesis regarding the pathogenesis of lung fibrosis indicated that a chronic inflammatory process may activate the fibrotic response through an anti-inflammatory mechanism disorder and a persisting exposure to an injurious antigen, thus resulting in the migration of hyperproliferative fibroblasts and subsequent production of the extracellular matrix (19). However, current evidence suggests that the sequentially injured and abnormally activated alveolar epithelial cells (AECs) are sufficient to drive the fibrotic response. These injured AECs release certain types of mediators, including transforming growth factor- $\beta$, which are able to induce the formation of fibroblast foci through the epithelial to mesenchymal transition (EMT) as well as the proliferation and transformation of residential mesenchymal cells (20).

EGFR, a cell surface receptor, is activated by binding to ligands, including the epidermal growth factor and transforming growth factor $\alpha$ (TGF- $\alpha$ ). Activated tyrosine kinase through the EGFR regulates cell growth, apoptosis and differentiation (21). EGFR is a member of the ErbB family, which includes EGFR (ErbB-1), HER2/c-neu (ErbB-2), Her 3 (ErbB-3) and Her 4 (ErbB-4) (22). Previous studies have demonstrated that EGFR and TGF- $\alpha$ played an important role in the development of lung fibrosis $(23,24)$. Alveolar type II cells in fibrotic lung tissues express higher levels of EGFR in comparison to cells in normal lung tissue, thus resulting in focal hyperplasia of alveolar epithelial cells. This indicates that epithelial regeneration through the EGFR-mediated pathways may be a potential mechanism for recovery from lung injury $(25,26)$. Therefore, EGFR inhibition induced by EGFR-TKIs is suggested to impair the ability of the type II cells to proliferate in order to regenerate the epithelial cells and augment lung fibrosis. TGF- $\alpha$ is a ligand that binds to EGFR and functions as a mitogen, which encourages cultured epithelial cells, fibroblasts and endothelial cells to proliferate. TGF- $\alpha$ has protective roles against lung injury, which include the attenuation of inflammation and the reduction of pulmonary edema (24). The blockade of EGFR-dependent phosphorylation by EGFR-TKIs inhibits EGFR-mediated signaling, resulting in the impairment of these protective roles by TGF- $\alpha$ and the exacerbation of lung fibrosis (27).

\section{Lung metabolic function}

The lung is extensively affected by exposure to internal agents in the systemic circulation as well as exposure to airborne particulates through the mouth and nose. Pulmonary vasculature receives the entire cardiac output. Similar to the liver, the lung is a metabolic organ containing chemical-metabolizing enzymatic systems, including P450 enzymes. Therefore, circulating agents, including toxicants, may be extracted from the plasma and become concentrated in the lung (28). Exposure to airborne particulates, including viruses, bacteria, vapors, fumes, dusts, gases and mists, are supposed to cause particulates-related lung diseases, e.g., lung fibrosis.

Although the average concentrations of P450 enzymes in the lung have been identified to be lower compared with that in the liver, alveolar type II epithelial cells and Clara cells appear to possess a higher P450 enzyme concentration (29). The high concentration of these enzymes in certain lung cell types appears to accelerate $\mathrm{P} 450$-dependent bioactivation, which subsequently generates highly toxic metabolic products. Lung exposure to these toxicants is able to induce epithelial injury, which may be associated to the development of lung-selective toxicity $(29,30)$.

Pyrrolizidine alkaloids (i.e., monocrotaline), included in various plants belonging to the Crotalaria and Sencicio genera, cause pulmonary hypertension in animal models (31). Pyrrolizidine alkaloids have been demonstrated to transit to alkylating agents through $\mathrm{P} 450$-dependent bioactivation in the liver, which is transported via the systemic circulation to the lung vasculature and may induce pulmonary arterial hypertension as well as pulmonary vascular remodeling (32). Similar to pyrrolizidine alkaloids, EGFR-TKIs are metabolized by P450 3A4 enzymes, which are predominantly present in the liver (33). Therefore, it is possible that toxic metabolic products from these TKIs, through P450-dependent bioactivation in the liver, may be transported to the lung and induce direct lung epithelial injury. Gefitinib and erlotinib are EGFR-TKIs and have similar chemical backbone structures. However, patients treated with gefitinib have a higher incidence of TKI-induced lung fibrosis compared with those treated with erlotinib (9). Gefitinib has been demonstrated to be more susceptible to P450-mediated metabolism in liver compared with erlotinib (34), which may contribute to the higher concentration of toxic metabolic products from gefitinib than erlotinib in the lung. The different susceptibilities between both TKIs to the liver metabolizing enzymes may therefore explain the different incidence of TKI-induced lung toxicity in the patients treated with gefitinib and erlotinib. 


\section{Conclusion}

Patients with any coincidental interstitial pneumonia, epithelial regeneration and restoration of the barrier function through EGFR-dependent epithelial cell proliferation may be stimulated by continuous unknown epithelial injury. Patients with predictive risk factors for the development of lung fibrosis (4) may have a reduced ability to regenerate and restore epithelial cells due to a poor functional status from cancer development. A decrease in pulmonary epithelial cell regeneration through the blockade of EGFR-dependent phosphorylation may play an important role in the development of EGFR-TKIs lung-selective toxicity.

The unique environment of the lung, where epithelial cells are exposed to internal agents and airborne particulates, may induce and/or accelerate the lung-specific toxicity induced by EGFR-TKIs.

\section{Acknowledgements}

This study was supported by research grants from the Respiratory Failure Research Group and the Cardiovascular Diseases Research Group (9-19,22-33) from the Ministry of Health, Labor and Welfare (Japan), and a Grant-in-Aid for Scientific Research (Category C; Grant No. 22590851) from the Japanese Ministry of Education and Science, and the Takeda Science Foundation.

\section{References}

1. Inoue A, Saijo Y, Maemondo M, et al: Severe acute interstitial pneumonia and gefitinib. Lancet 361: 137-139, 2003.

2. Makris D, Scherpereel A, Copin M, et al: Fatal interstitial lung disease associated with oral erlotinib therapy for lung cancer. BMC Cancer 7: 150, 2007.

3. Liu V, White D and Zakowski M, et al: Pulmonary toxicity associated with erlotinib. Chest 132: 1042-1044, 2007.

4. Ando M, Okamoto I, Yamamoto N, et al: Predictive factors for interstitial lung disease, antitumor response, and survival in non-small-cell lung cancer patients treated with gefitinib. J Clin Oncol 24: 2549-2556, 2006.

5. Chou CL, Ko HW, Wang CW, Yu CT, Kuo HP and Huang CD: Erlotinib-associated near-fatal interstitial pneumonitis in a patient with relapsed lung adenocarcinoma. Chang Gung Med J 33: 100-105, 2010.

6. Vahid B and Esmaili A: Erlotinib-associated acute pneumonitis: report of two cases. Can Respir J Can Thorac Soc 14: 167-170, 2007.

7. Del Castillo Y, Espinosa P, Bodí F, et al: Interstitial lung disease associated to erlotinib treatment: a case report. Cases J 3: 59, 2010.

8. Dimopoulou I, Bamias A, Lyberopoulos P and Dimopoulos MA: Pulmonary toxicity from novel antineoplastic agents. Ann Oncol 17: 372-379, 2006.

9. Peerzada MM, Spiro TP and Daw HA: Pulmonary toxicities of tyrosine kinase inhibitors. Clin Adv Hematol Oncol 9: 824-836, 2011.

10. Min JH, Lee HY, Lim H, et al: Drug-induced interstitial lung disease in tyrosine kinase inhibitor therapy for non-small cell lung cancer: a review on current insight. Cancer Chemother Pharmacol 68: 1099-1109, 2011.

11. Mok TS, Wu YL, Thongprasert S, et al: Gefitinib or carboplatin-paclitaxel in pulmonary adenocarcinoma. N Engl J Med 361: 947-957, 2009.
12. Maemondo M, Inoue A, Kobayashi K, et al: Gefitinib or chemotherapy for non-small-cell lung cancer with mutated EGFR. N Engl J Med 362: 2380-2388, 2010.

13. Lynch TJ, Bell DW, Sordella R, et al: Activating mutations in the epidermal growth factor receptor underlying responsiveness of non-small-cell lung cancer to gefitinib. N Engl J Med 350: 2129-2139, 2004.

14. Paez JG, Janne PA, Lee JC, et al: EGFR mutations in lung cancer: correlation with clinical response to gefitinib therapy. Science 304: 1497-500, 2004.

15. Sordella R, Bell DW, Haber DA and Settleman J: Gefitinibsensitizing EGFR mutations in lung cancer activate anti-apoptotic pathways. Science 305: 1163-1167, 2004.

16. Cohen MH, Johnson JR and Pazdur R: U.S. food and drug administration drug approval summary: conversion of imatinib mesylate (sti571; gleevec) tablets from accelerated approval to full approval. Clin Cancer Res 11: 12-19, 2005.

17. Takeuchi $\mathrm{K}$ and Ito F: Receptor tyrosine kinases and targeted cancer therapeutics. Biol Pharm Bull 34: 1774-1780, 2011.

18. Richeldi L, Costabel U, Selman M, et al: Efficacy of a tyrosine kinase inhibitor in idiopathic pulmonary fibrosis. N Engl J Med 365: 1079-1087, 2011.

19. Keogh BA and Crystal RG: Alveolitis: the key to the interstitial lung disorders. Thorax 37: 1-10, 1982.

20. King TE Jr, Pardo A and Selman M: Idiopathic pulmonary fibrosis. Lancet 378: 1949-1961, 2011.

21. Chen WS, Lazar CS, Poenie M, et al: Requirement for intrinsic protein tyrosine kinase in the immediate and late actions of the EGF receptor. Nature 328: 820-823, 1987.

22. Yarden Y and Sliwkowski MX: Untangling the ErbB signalling network. Nat Rev Mol Cell Biol 2: 127-137, 2001.

23. Suzuki H, Aoshiba K, Yokohori N and Nagai A: Epidermal growth factor receptor tyrosine kinase inhibition augments a murine model of pulmonary fibrosis. Cancer Res 63: 5054-5059, 2003.

24. Madtes DK, Busby HK, Strandjord TP and Clark JG: Expression of transforming growth factor-alpha and epidermal growth factor receptor is increased following bleomycin-induced lung injury in rats. Am J Respir Cell Mol Biol 11: 540-551, 1994.

25. Selman M, King TE and Pardo A; American Thoracic Society; European Respiratory Scoiety; American College of Chest Physicians: Idiopathic pulmonary fibrosis: prevailing and evolving hypotheses about its pathogenesis and implications for therapy. Ann Intern Med 134: 136-151, 2001.

26. Baughman RP, Lower EE, Miller MA, Bejarano PA and Heffelfinger SC: Overexpression of transforming growth factor-alpha and epidermal growth factor-receptor in idiopathic pulmonary fibrosis. Sarcoidosis Vasc Diffuse Lung Dis 16: 57-61, 1999

27. Hardie WD, Prows DR, Piljan-Gentle A, et al: Dose-related protection from nickel-induced lung injury in transgenic mice expressing human transforming growth factor-alpha. Am J Respir Cell Mol Biol 26: 430-437, 2002.

28. Smith BR and Brian WR: The role of metabolism in chemical-induced pulmonary toxicity. Toxicol Pathol 19: 470-481, 1991.

29. Guengerich FP: Purification and characterization of xenobiotic-metabolizing enzymes from lung tissue. Pharmacol Ther 45: 299-308, 1990.

30. Devereux TR, Domin BA and Philpot RM: Xenobiotic metabolism by isolated pulmonary cells. Pharmacol Ther 41: 243-256, 1989.

31. Roth RA and Reindel JF: Lung vascular injury from monocrotaline pyrrole, a putative hepatic metabolite. Adv Exp Med Biol 283: 477-487, 1991.

32. Williams DE, Reed RL, Kedzierski G, Dannan GA, Guengerich FP and Buhler DR: Bioactivation and detoxication of the pyrrolizidine alkaloid senecionine by cytochrome P-450 enzymes in rat liver. Drug Metab Dispos 17: 387-392, 1989.

33. McKillop D, McCormick AD, Millar A, Miles GS, Phillips PJ and Hutchison M: Cytochrome P450-dependent metabolism of gefitinib. Xenobiotica 35: 39-50, 2005.

34. Li J, Zhao M, He P, Hidalgo M and Baker SD: Differential metabolism of gefitinib and erlotinib by human cytochrome P450 enzymes. Clin Cancer Res 3731-3737, 2007. 